\title{
Hybrid multi-Bernoulli CPHD filter for superpositional sensors
}

\author{
Santosh Nannuru and Mark Coates \\ McGill University, Montreal, Canada
}

\begin{abstract}
We propose, for the superpositional sensor scenario, a hybrid between the multi-Bernoulli filter and the cardinalized probability hypothesis density (CPHD) filter. We use a multi-Bernoulli random finite set (RFS) to model existing targets and we use an independent and identically distributed cluster (IIDC) RFS to model newborn targets and targets with low probability of existence. Our main contributions are providing the update equations of the hybrid filter and identifying computationally tractable approximations. We achieve this by defining conditional probability hypothesis densities (PHDs), where the conditioning is on one of the targets having a specified state. The filter performs an approximate Bayes update of the conditional PHDs. In parallel, we perform a cardinality update of the IIDC RFS component in order to estimate the number of newborn targets. We provide an auxiliary particle filter based implementation of the proposed filter and compare it with CPHD and multi-Bernoulli filters in a simulated multitarget tracking application.
\end{abstract}

Keywords: Random finite sets, CPHD filter, multi-Bernoulli filter, hybrid multi-Bernoulli CPHD filter

\section{INTRODUCTION}

The random finite set approach has been successfully used to perform multitarget tracking and estimation in recent years. The idea was first developed by Mahler ${ }^{1,2}$ and subsequently many different filters have been derived using this framework, including the probability hypothesis density (PHD) filter, the CPHD (Cardinalized PHD) filter and the multi-Bernoulli filter. Traditionally the filters have been developed under somewhat restrictive assumptions about the measurement devices; Mahler has called these "standard sensors". ${ }^{3}$ The observation model we consider deviates from this sensor class, and the associated sensors have been called superpositional sensors. ${ }^{3}$ In contrast to the standard sensor assumption, in which each target is associated with at most one measurement, in the superpositional sensor model, each target can contribute to any number of measurements, but the contributions must be additive (or superpositional). This model is suited to a number of different sensors including direction-of-arrival sensors for linear antenna arrays, ${ }^{4}$ multipath channels in MIMO-OFDM channels, ${ }^{5}$ acoustic amplitude sensors ${ }^{6}$ and radio frequency tomographic tracking sensor systems. ${ }^{7}$

In past work addressing the development of moment-based filters for the superpositional sensor model, Thouin et al. derived the PHD filter equations ${ }^{8}{ }^{*}$ and Mahler and El-Fallah extended the derivation to the CPHD filter. ${ }^{10}$ Nannuru et al. presented implementations of the PHD and CPHD filters for superpositional sensors based on the auxiliary particle filter approach. ${ }^{11}$ Hauschildt et al. derived a closed form Gaussian mixture implementation of the CPHD filter for superpositional sensors. ${ }^{12}$ Nannuru and Coates proposed a multi-Bernoulli filter for superpositional sensors ${ }^{13}$ and presented a particle filter implementation. ${ }^{14}$

The multi-Bernoulli filter is able to provide more accurate target location estimates than the CPHD filter, but its cardinality estimates are less robust to the arrival of new targets and the disappearance of existing targets. To address this issue, we propose in this paper a hybrid between the multi-Bernoulli filter and the CPHD filter. In this hybrid approach the new targets are modeled using the independent and identically distributed cluster (IIDC) RFS while the existing targets are modeled using the multi-Bernoulli RFS. To the best of our knowledge, combination of the multi-Bernoulli filter with the CPHD filter has not been proposed in the literature (neither for the standard sensor model nor the superpositional sensor model). Williams ${ }^{15,16}$ proposed a hybrid between the multi-Bernoulli filter and the PHD filter for multitarget tracking applications. Williams uses a Poisson RFS to

\footnotetext{
${ }^{*}$ An error in the main update equation of this filter was corrected in an errata $;^{9}$ the correct equations were also presented later. ${ }^{10,11}$
} 
model new targets and targets with low probability of existence. Pollard et al. developed a hybrid combination of the multiple hypothesis tracking (MHT) filter and the Gaussian mixture CPHD (GM-CPHD) filter. ${ }^{17}$ The GMCPHD filter provides a robust cardinality estimate of the multitarget state which is complemented by accurate state estimates from the MHT filter. Panta et al. proposed a similar hybrid approach combining the MHT and PHD filters; ${ }^{18}$ in their work the PHD filter is used as a clutter filter to gate the input for the MHT filter.

The paper is organized as follows: Section 1.1 formulates the multitarget tracking problem, and Section 1.2 explains the superpositional sensor model. Section 2 provides a brief background on random finite set theory and presents the concept of the conditional PHD. Section 3 presents the prediction and update steps of the proposed hybrid multi-Bernoulli CPHD filter, and Section 4 outlines a particle filter based implementation of the proposed filter. Section 5 describes a numerical analysis of the filter implementation and conclusions are drawn in Section 6.

\subsection{Problem formulation}

The superpositional sensor model is relevant for many different state estimation problems. ${ }^{4-7}$ The proposed hybrid multi-Bernoulli filter can be used for state tracking and estimation in all these applications. For this paper we develop the filter equations for a multitarget tracking problem and use the same framework to demonstrate the filter performance in simulations. If we have $n_{k} \geq 0$ targets present at time step $k$ then the multitarget state to be tracked is represented by the set $X_{k}=\left\{\mathbf{x}_{k, 1}, \mathbf{x}_{k, 2}, \ldots, \mathbf{x}_{k, n_{k}}\right\}$ with $\mathbf{x}_{k, i} \in R^{n_{\mathbf{x}}} \forall i$. The evolution of the target state over time is given by the transition kernel $t\left(\mathbf{x}_{k, i} \mid \mathbf{x}_{k-1, i}, \mathbf{u}_{k}\right)$ where $\mathbf{u}_{k}$ is a Gaussian noise vector. The motion of each target is assumed to be independent of the other targets. The multitarget state $X_{k}$ is related to the observation $\mathbf{z}_{k} \in R^{n_{\mathbf{z}}}$ by the likelihood function $h_{\mathbf{z}_{k}}\left(X_{k}\right)$. If we define $Z^{[k]}=\left[\mathbf{z}_{1}, \mathbf{z}_{2}, \ldots, \mathbf{z}_{k}\right]$, the tracking problem requires estimation of the posterior multitarget distribution $p\left(X_{k} \mid Z^{[k]}\right)$ for each time step $k$.

\subsection{Superpositional sensor model}

We now briefly discuss the superpositional sensor model. The likelihood function is said to have a superpositional form if it can be written as follows

$$
\begin{aligned}
h_{\mathbf{z}_{k}}\left(X_{k}\right) & =h_{\mathbf{z}_{k}}\left(r\left(X_{k}\right)\right) \\
& =h_{\mathbf{z}_{k}}\left(\sum_{\mathbf{x} \in X_{k}} g(\mathbf{x})\right)
\end{aligned}
$$

where the function $r$ is defined over sets and the function $g$ is defined over the single target state space. Both the functions $r$ and $g$ map to vectors with real elements and can be non-linear. If we model the sensor noise as Gaussian with zero mean and covariance matrix $\Sigma_{r}$, the above likelihood can be simplified and written as

$$
h_{\mathbf{z}_{k}}\left(X_{k}\right)=\mathcal{N}_{\Sigma_{r}}\left(\mathbf{z}_{k}-r\left(X_{k}\right)\right) .
$$

where $\mathcal{N}_{\Sigma}(\mathbf{z})$ denotes the Gaussian density function with zero mean and covariance matrix $\Sigma$ evaluated at $\mathbf{z}$.

\section{RANDOM FINITE SETS}

In the random finite set framework we can model the multitarget state and the observations as realizations of random sets. This allows us to capture the randomness in both the dimension and elements of the set. A RFS is completely specified by its multitarget density function $f(W)$ which integrates to one. The cardinality distribution function of the random set is given by $\pi(n)=\operatorname{Prob}(|W|=n)$, where $|W|$ denotes the cardinality of the set W. An important and useful quantity, analogous to the expectation of a random vector, is the probability hypothesis density (PHD) function associated with the random finite set. The PHD function $D(\mathbf{x})$ is defined as

$$
D(\mathbf{x})=\int f(\{\mathbf{x}\} \cup W) \delta W
$$

Examples of some random finite sets are the Poisson RFS, the independent and identically distributed cluster (IIDC) RFS, the Bernoulli RFS and the multi-Bernoulli RFS. An independent and identically distributed cluster 
RFS can be realized by sampling the number of elements from the discrete cardinality distribution $\pi^{c}(n)$ and then independently sampling all the elements from the density function $q_{c}(\mathbf{x})$. Let $\mu_{c}$ denote the mean of the cardinality distribution $\pi^{c}(n)$. The PHD function of the IIDC RFS can be calculated to be

$$
D^{c}(\mathbf{x})=\int f(\{\mathbf{x}\} \cup W) \delta W=\mu_{c} \cdot q_{c}(\mathbf{x}) .
$$

A multi-Bernoulli RFS is the union of independent Bernoulli random finite sets. Each of the Bernoulli random finites sets is specified by its existence probability $r_{i}$ and the state density function $q_{i}(\mathbf{x})$. The PHD function of the multi-Bernoulli RFS is given by (Ex. 91, Chap. $16,{ }^{2}$ ),

$$
D^{m b}(\mathbf{x})=\sum_{i=1}^{N} r_{i} \cdot q_{i}(\mathbf{x}) .
$$

\subsection{Conditional PHD}

A conditional PHD can be defined when a random finite set is a union of multiple random finite sets. For example, let the RFS $\chi$ be the union of the random finites sets $\chi_{A}$ and $\chi_{B}$. Let $f(W)$ be the multitarget density of the RFS $\chi$. Then the conditional PHDs corresponding to the components $\chi_{A}$ and $\chi_{B}$ of $\chi$ are defined as

$$
\begin{aligned}
& \mathcal{D}^{A}(\mathbf{x})=\int f(\{\mathbf{x}\} \cup W \mid \mathbf{x} \leftarrow A) \delta W \\
& \mathcal{D}^{B}(\mathbf{x})=\int f(\{\mathbf{x}\} \cup W \mid \mathbf{x} \leftarrow B) \delta W
\end{aligned}
$$

The conditioning event $(\mathbf{x} \leftarrow A)$ implies that if $\mathbf{x}$ is a member of the multitarget state, then element $\mathbf{x}$ is generated by the random finite set $\chi_{A}$. Similarly we can define the conditioning event $(\mathbf{x} \leftarrow B)$.

If $D^{A}(\mathbf{x})$ and $D^{B}(\mathbf{x})$ are the PHD functions of RFSs $\chi_{A}$ and $\chi_{B}$ respectively then we can show that

$$
\mathcal{D}^{A}(\mathbf{x})=D^{A}(\mathbf{x}) \quad \text { and } \quad \mathcal{D}^{B}(\mathbf{x})=D^{B}(\mathbf{x})
$$

The above result is proved in the technical report. ${ }^{19}$

\subsection{Conditional PHD update}

Let $\mathcal{D}_{k+1 \mid k}^{A}(\mathbf{x})$ and $\mathcal{D}_{k+1}^{A}(\mathbf{x})$ denote the predicted and posterior conditional PHD corresponding to the RFS component $\chi_{A}$ of $\chi$ at time $k+1$. The conditional PHD update equation relates these two quantities by applying Bayes' rule. It can be shown that

$$
\mathcal{D}_{k+1}^{A}(\mathbf{x})=\mathcal{D}_{k+1 \mid k}^{A}(\mathbf{x}) \frac{\int h_{\mathbf{z}_{k+1}}(\{\mathbf{x}\} \cup W) \times f_{k+1 \mid k}^{\bar{A}}(W) \delta W}{\int h_{\mathbf{z}_{k+1}}(W) \times f_{k+1 \mid k}(W) \delta W}
$$

where $f_{k+1 \mid k}(W)$ is the predicted multitarget distribution. The multitarget distribution $f_{k+1 \mid k}^{\bar{A}}(W)$ is defined as

$$
f_{k+1 \mid k}^{\bar{A}}(W)=\frac{f_{k+1 \mid k}(\{\mathbf{x}\} \cup W \mid \mathbf{x} \leftarrow A)}{\mathcal{D}_{k+1 \mid k}^{A}(\mathbf{x})} .
$$

The derivation of the above update equation is provided in the technical report. ${ }^{19}$ We will use the above result for the special case of union of independent IIDC RFS and multi-Bernoulli RFS to derive the update equations for the hybrid multi-Bernoulli CPHD filter.

\section{HYBRID MULTI-BERNOULLI CPHD FILTER}

The multi-Bernoulli filter has one density function for each target allowing accurate state estimation and easy track management but does not have a robust cardinality estimator. The CPHD filter can provide robust cardinality estimates but uses only a single density function to model all the target states. In the hybrid approach we combine these filters so that existing targets are represented by Bernoulli components while the new targets are modeled using an IIDC RFS component. 


\subsection{Prediction step}

The prediction step propagates the Bernoulli components from the previous time step using the motion model for surviving targets and initializes the IIDC component using the target birth model. Let the posterior Bernoulli components at time $k$ be parametrized by $\left\{r_{k, i}, q_{k, i}(\mathbf{x})\right\}_{i=1}^{N_{k}}$. Denote the predicted Bernoulli components at time $k+1$ as $\left\{r_{i}, q_{i}(\mathbf{x})\right\}_{i=1}^{N_{k}}$. Then we have the relations ${ }^{2,20}$

$$
\begin{aligned}
r_{i} & =r_{k, i} \times\left\langle q_{k, i}, p_{s}\right\rangle, \\
q_{i}(\mathbf{x}) & =\frac{\left\langle t_{k+1 \mid k}(\mathbf{x} \mid \cdot), q_{k, i} p_{s}\right\rangle}{\left\langle q_{k, i}, p_{s}\right\rangle} ; \quad \forall i=1 \ldots N_{k}
\end{aligned}
$$

where $p_{s}(\mathbf{x})$ is the target survival probability, $t_{k+1 \mid k}(\mathbf{x} \mid \cdot)$ is the Markov transition kernel and $\langle a, b\rangle$ is the scalar product defined as $\langle a, b\rangle=\int a(\mathbf{x}) b(\mathbf{x}) d \mathbf{x}$. Note that the number of Bernoulli components does not change in the prediction step. To account for the new born targets an independent IIDC component is initialized. Let its cardinality distribution function be $\pi_{k+1 \mid k}^{c}(n)$ and its state density function be $q_{c}(\mathbf{x})=q_{c, k+1 \mid k}(\mathbf{x})$. Thus the predicted multitarget state is union of an independent IIDC component and a multi-Bernoulli component.

\subsection{Update step}

The conditional PHD update equation in (9) is not analytically implementable because of the set integrals involved. It has been shown that ${ }^{19}$ for the case of superpositional sensor model as given in equations (1) and (2), under the Gaussian sensor noise assumption, the update equation can be approximated as

$$
\mathcal{D}_{k+1}^{A}(\mathbf{x}) \approx \mathcal{D}_{k+1 \mid k}^{A}(\mathbf{x}) \frac{\mathcal{N}_{\Sigma_{r}+\Sigma_{k+1}^{\bar{A}}}\left(\mathbf{z}_{k+1}-g(\mathbf{x})-\mu_{k+1}^{\bar{A}}\right)}{\mathcal{N}_{\Sigma_{r}+\Sigma_{k+1}}\left(\mathbf{z}_{k+1}-\mu_{k+1}\right)}
$$

where the parameters $\mu_{k+1}, \mu_{k+1}^{\bar{A}}, \Sigma_{k+1}$ and $\Sigma_{k+1}^{\bar{A}}$ can be found using the quadratic version of Campbell's theorem. ${ }^{10,11}$ The update stage of the hybrid multi-Bernoulli CPHD filter propagates the conditional PHD for each of the Bernoulli components, conditional PHD of the IIDC component and the cardinality distribution of the IIDC component. Thus the posterior state is also modeled as the union of an independent IIDC component and a multi-Bernoulli component. The IIDC component is later approximated by a multi-Bernoulli component. Applying the result in equation (13) to update the conditional PHD of the Bernoulli component we get

$$
r_{i}^{\prime} \cdot q_{i}^{\prime}(\mathbf{x}) \approx r_{i} \cdot q_{i}(\mathbf{x}) \frac{\mathcal{N}_{\Sigma_{r}+\Sigma_{k+1}^{\bar{i}}}\left(\mathbf{z}_{k+1}-g(\mathbf{x})-\mu_{k+1}^{\bar{i}}\right)}{\mathcal{N}_{\Sigma_{r}+\Sigma_{k+1}}\left(\mathbf{z}_{k+1}-\mu_{k+1}\right)}
$$

where,

$$
\begin{aligned}
\mu_{k+1}=\sum_{i=1}^{N_{k}} r_{i} \cdot s_{i}+\mu_{c} \cdot s_{c} \\
\Sigma_{k+1}=\sum_{i=1}^{N_{k}}\left(r_{i} \cdot v_{i}-r_{i}^{2} \cdot s_{i} s_{i}^{T}\right)+\mu_{c} \cdot v_{c}-\left(\mu_{c}^{2}-a\right) \cdot s_{c} s_{c}^{T} \\
\mu_{k+1}^{\bar{i}}=\mu_{k+1}-r_{i} \cdot s_{i}, \quad \Sigma_{k+1}^{\bar{i}}=\Sigma_{k+1}-\left(r_{i} \cdot v_{i}-r_{i}^{2} \cdot s_{i} s_{i}^{T}\right) \\
s_{i}=\left\langle q_{i}, g\right\rangle, \quad v_{i}=\left\langle q_{i}, g g^{T}\right\rangle, \quad s_{c}=\left\langle q_{c}, g\right\rangle, \quad v_{c}=\left\langle q_{c}, g g^{T}\right\rangle \\
a=\sum_{n} n(n-1) \pi_{k+1 \mid k}^{c}(n)
\end{aligned}
$$

The derivation of the above result and the expressions for the various parameters involved are provided in the technical report. ${ }^{19}$ Similarly, applying the result of equation (13) to the conditional PHD of IIDC RFS component 
leads to the following approximate update:

$$
\mu_{c}^{\prime} \cdot q_{c}^{\prime}(\mathbf{x}) \approx \mu_{c} \cdot q_{c}(\mathbf{x}) \frac{\mathcal{N}_{\Sigma_{r}+\Sigma_{k+1}^{\bar{c}}}\left(\mathbf{z}_{k+1}-g(\mathbf{x})-\mu_{k+1}^{\bar{c}}\right)}{\mathcal{N}_{\Sigma_{r}+\Sigma_{k+1}}\left(\mathbf{z}_{k+1}-\mu_{k+1}\right)}
$$

where

$$
\begin{aligned}
\mu_{k+1}^{\bar{c}} & =\sum_{j=1}^{N_{k}} r_{j} \cdot s_{j}+\frac{a}{\mu_{c}} \cdot s_{c} \\
\Sigma_{k+1}^{\bar{c}} & =\sum_{j=1}^{N_{k}}\left(r_{j} \cdot v_{j}-r_{j}^{2} \cdot s_{j} s_{j}^{T}\right)+\frac{a}{\mu_{c}} v_{c}-\left(\frac{a^{2}}{\mu_{c}^{2}}-\frac{b}{\mu_{c}}\right) s_{c} s_{c}^{T} \\
b & =\sum_{n} n(n-1)(n-2) \pi_{k+1 \mid k}^{c}(n)
\end{aligned}
$$

where $\mu_{k+1}$ and $\Sigma_{k+1}$ are as given in equations (15) and (16) respectively. The derivation of the above result is provided in the technical report. ${ }^{19}$

To complete the update step we also need to propagate the cardinality distribution of the IIDC component. The cardinality update equation is given as

$$
\pi_{k+1}^{c}(n) \approx \pi_{k+1 \mid k}^{c}(n) \frac{\mathcal{N}_{\Sigma_{r}+\Sigma_{k+1}^{c, n}}\left(\mathbf{z}_{k+1}-\mu_{k+1}^{c, n}\right)}{\mathcal{N}_{\Sigma_{r}+\Sigma_{k+1}}\left(\mathbf{z}_{k+1}-\mu_{k+1}\right)}
$$

where

$$
\begin{aligned}
\mu_{k+1}^{c, n} & =\sum_{i=1}^{N_{k+1 \mid k}} r_{i} \cdot s_{i}+n \cdot s_{c} \\
\Sigma_{k+1}^{c, n} & =\sum_{i=1}^{N_{k+1 \mid k}}\left(r_{i} \cdot v_{i}-r_{i}^{2} \cdot s_{i} s_{i}^{T}\right)+n\left(v_{c}-s_{c} s_{c}^{T}\right)
\end{aligned}
$$

where $\mu_{k+1}$ and $\Sigma_{k+1}$ are as given in equations (15) and (16) respectively. The derivation of the above result is provided in the technical report. ${ }^{19}$

\section{PARTICLE FILTER IMPLEMENTATION}

We now present an auxiliary particle filter based implementation of the hybrid multi-Bernoulli CPHD filter. The pseudocode for the filter implementation is provided in Figure 1. Each of the conditional PHD terms to be propagated is represented using a set of weighted particles. Thus we have one particle filter for each of the Bernoulli components and one particle filter for the IIDC component. In the prediction step the conditional PHD of the Bernoulli components is propagated using the motion model of surviving targets whereas the conditional PHD of the IIDC component is initialized using the target birth model. For the update step the particle weights are updated using equations (20) and (14). We perform the update in two stages so that the new born targets are accurately identified. After the update stage, the IIDC conditional PHD is approximated using multiple Bernoulli conditional PHDs. The number of Bernoulli components added depends on the maximum a posteriori (MAP) estimate obtained from the updated posterior IIDC cardinality distribution. Bernoulli tracks with low existence probability are pruned. Gating is performed to avoid multiple Bernoulli components representing a single target and is achieved by terminating new tracks appearing within close vicinity of existing Bernoulli components. 


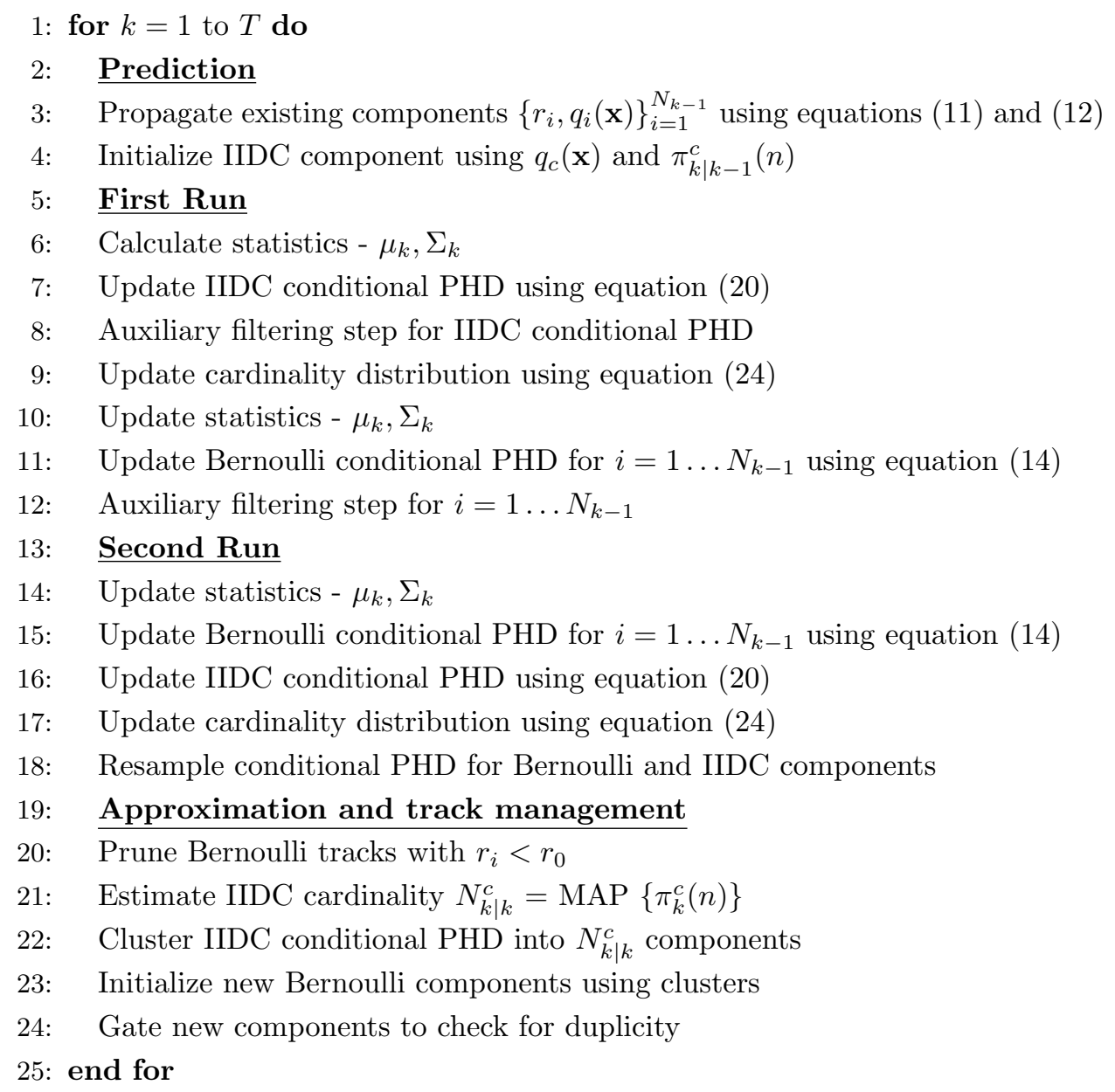

Figure 1: Pseudo-code for auxiliary particle filter implementation of hybrid multiBernoulli CPHD filter.

\section{NUMERICAL SIMULATIONS}

In this section we compare the auxiliary particle filter implementations of the CPHD filter, multi-Bernoulli filter (MBR) and hybrid multi-Bernoulli CPHD filter (MBR-CPHD). The implementations are used to perform tracking of multiple targets moving in a region monitored by radio frequency sensors. The motion of individual targets is assumed to be independent of one another and follows a linear Gaussian model. ${ }^{21}$ A single target state consists of its two position coordinates and two velocity coordinates in the plane of motion of targets.

The targets are moving within a region monitored by radio frequency $(\mathrm{RF})$ sensors. The RF sensor multitarget measurement model used has been proposed and empirically verified. ${ }^{22,23}$ In our experiments we simulate an RF sensor network of 20 sensor nodes which are uniformly distributed around a square region $20 \mathrm{~m} \times 20 \mathrm{~m}$ in dimension as shown in Figure 2a. There are 190 unique bidirectional links among the sensors giving rise to the measurement dimension of $n_{\mathbf{z}}=190$. The observation model parameters are $\phi=5, \sigma_{\lambda}=0.4$ and $\sigma_{r}^{2}=0.25$. Target births are assumed to be uniform within the monitoring region. Bernoulli tracks with existence probability less than $r_{0}=0.2$ are pruned and gating is performed with a $1 \mathrm{~m}$ gating radius. To account for target births 4 new Bernoulli components are added at each time step in implementation of the MBR filter. We use 1000 particles for each of the particle filter in our implementation.

We simulate two types of target track variations: (i) a fixed number of targets (6) are present in the monitoring region (Exp. 1); and (ii) the targets can appear and disappear over time with a maximum of 6 targets (Exp. 2). 
Figure 2a and Figure 2b show the target tracks we use for Exp. 1 and Exp. 2 respectively. The blue cross (x) indicates the starting location of the target. The variation of number of targets over time for Exp. 2 is shown in Figure 3a. Each simulation lasts for 35 time steps. Targets labeled number 5 and 6 in Figure 2b appear within the monitoring region at time steps 9 and 17 respectively. Target 6 disappears from the monitoring region at time step 24 .

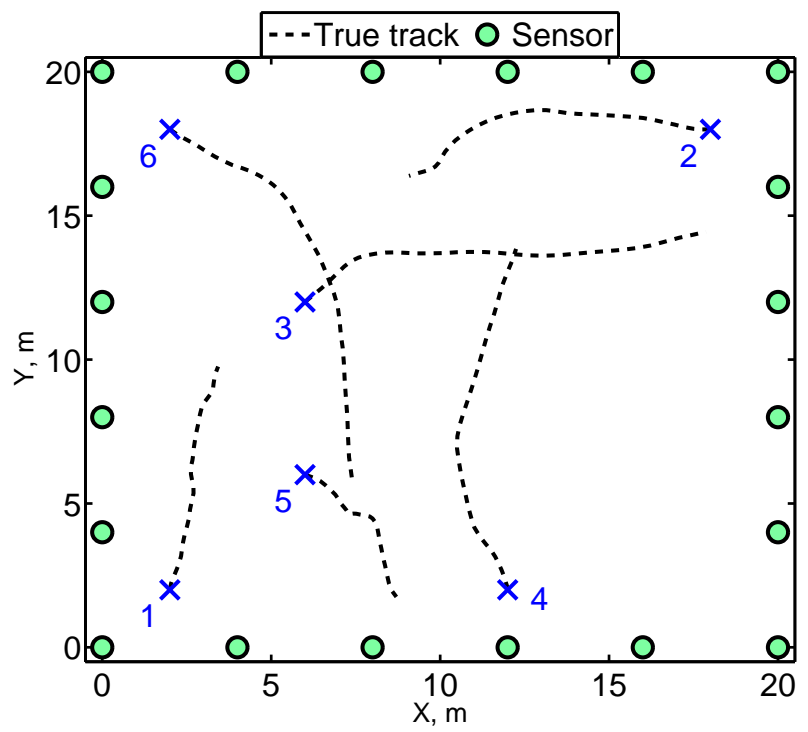

(a) Exp. 1 Target tracks

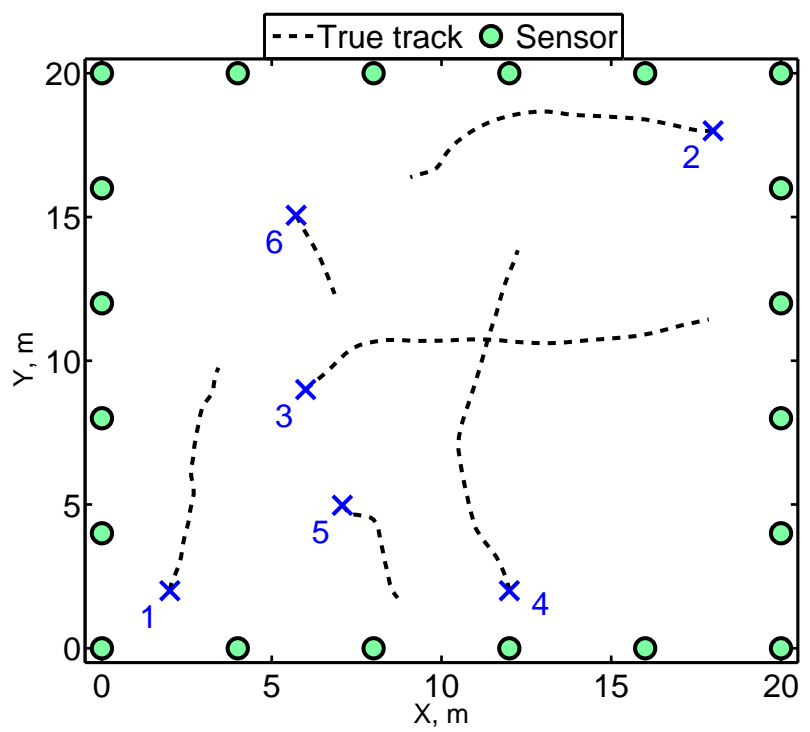

(b) Exp. 2 Target tracks

Figure 2: Tracks of targets for the two experiments

To compare the performance of different filters we need an objective error metric. We use the optimal subpattern assignment (OSPA) metric ${ }^{24}$ to compare the true and estimated multitarget states. The OSPA metric can compare sets of varying dimensionality and penalize errors in cardinality estimation using the cardinality penalty factor $c$. The multitarget state is estimated by calculating, for each Bernoulli component, the average of all the particles associated with that component after the resampling step.

For each of the above two experiments, 20 different measurement sequences are generated by changing the realisation of the noise and for each realisation the tracking algorithms are applied 5 times with different initialization each time. Thus the target tracks are processed for a total of $20 \times 5=100$ times and the average error is calculated over all the 100 runs. The average OSPA error calculations for Exp. 1 data are shown in Table 1 for three different values of cardinality penalty factor $c$. A higher value of $c$ implies a higher error penalty for each cardinality mistake made by the filter. The CPHD filter and the MBR-CPHD filter both perform better than the plain MBR filter for all values of $c$. For low values of $c$ the MBR-CPHD filter performs slightly better than the CPHD filter. This analysis implies that the cardinality estimate of the CPHD filter is very robust whereas the hybrid MBR-CPHD filter can provide better state estimates. The average processing times of the different algorithms per time step, when using a desktop computer, have been reported in Table 1. The hybrid approach is the fastest; it does not have to do the computationally demanding clustering at each time step like the CPHD filter, nor must it process multiple redundant Bernoulli components to account for target births like the multi-Bernoulli filter.

Table 1: Mean and standard deviation of the OSPA error and average processing times for Exp. 1.

\begin{tabular}{|c|c|c|c||c|}
\hline Algorithm & \multicolumn{2}{|c|}{ OSPA error (Mean \pm SD) } & Processing time (s) \\
\hline & $c=0.5$ & $c=1$ & $c=1.5$ & \\
\hline CPHD & $0.12 \pm 0.01$ & $0.12 \pm 0.01$ & $\mathbf{0 . 1 2} \pm \mathbf{0 . 0 1}$ & 1.00 \\
\hline MBR & $0.12 \pm 0.02$ & $0.15 \pm 0.05$ & $0.17 \pm 0.07$ & 0.94 \\
\hline MBR-CPHD & $\mathbf{0 . 1 1} \pm \mathbf{0 . 0 1}$ & $\mathbf{0 . 1 1} \pm \mathbf{0 . 0 2}$ & $0.12 \pm 0.03$ & 0.66 \\
\hline
\end{tabular}


An average OSPA error analysis for the Exp. 2 data is shown in Table 2. The MBR filter has relatively higher error compared to the previous experiment and the error increases significantly with the increase in value of $c$ indicating that there are frequent cardinality errors. The MBR-CPHD filter performs better than the CPHD filter at all values of $c$. Thus the hybrid MBR-CPHD filter is able to inherit the robust cardinality estimate from the CPHD filter and accurate state estimates from the MBR filter. The average processing time of the algorithms are provided in Table 2.

Table 2: Mean and standard deviation of the OSPA error and average processing times for Exp. 2.

\begin{tabular}{|c|c|c|c||c|}
\hline Algorithm & \multicolumn{2}{|c|}{ OSPA error (Mean \pm SD) } & Processing time (s) \\
\hline & $c=0.5$ & $c=1$ & $c=1.5$ & \\
\hline CPHD & $0.13 \pm 0.01$ & $0.13 \pm 0.01$ & $0.13 \pm 0.01$ & 0.82 \\
\hline MBR & $0.14 \pm 0.03$ & $0.19 \pm 0.07$ & $0.23 \pm 0.12$ & 0.87 \\
\hline MBR-CPHD & $\mathbf{0 . 1 1} \pm \mathbf{0 . 0 1}$ & $\mathbf{0 . 1 2} \pm \mathbf{0 . 0 2}$ & $\mathbf{0 . 1 2} \pm \mathbf{0 . 0 2}$ & 0.59 \\
\hline
\end{tabular}

To further analyse the simulation results for Exp. 2 a box-and-whisker plot of the OSPA error as the simulation progresses is shown in Figure 3b for the cardinality penalty factor $c=0.5$. The cardinality variation in Exp.2 is shown in Figure 3a. At time steps 9 and 17 new targets appear in the monitoring region and we note that the MBR filter shows a large variation in its OSPA error at and after those time steps due to cardinality estimation errors. The hybrid MBR-CPHD filter has lower median error when compared to the CPHD filter at most time steps. A larger number of outliers can be seen in the plot for the MBR and MBR-CPHD filters when compared to the CPHD filter which is also reflected as increased standard deviation in Table 2.

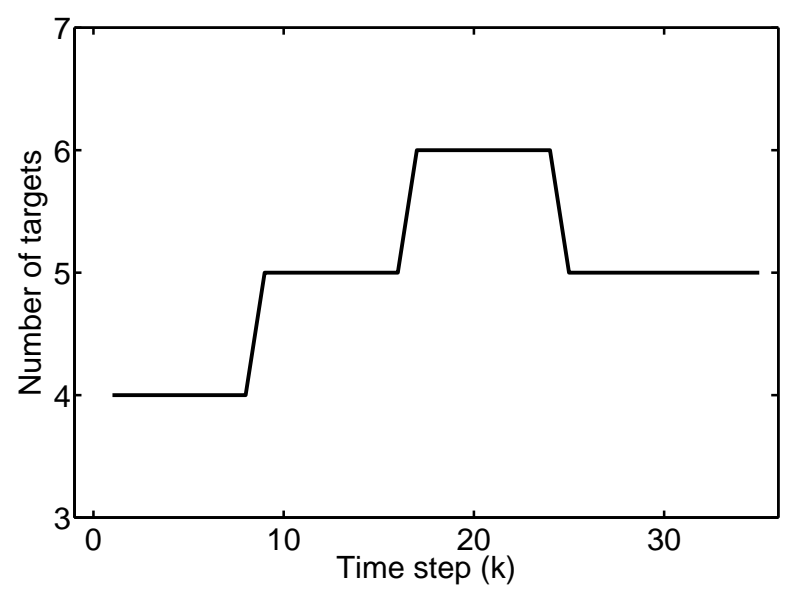

(a) Exp. 2 Number of targets

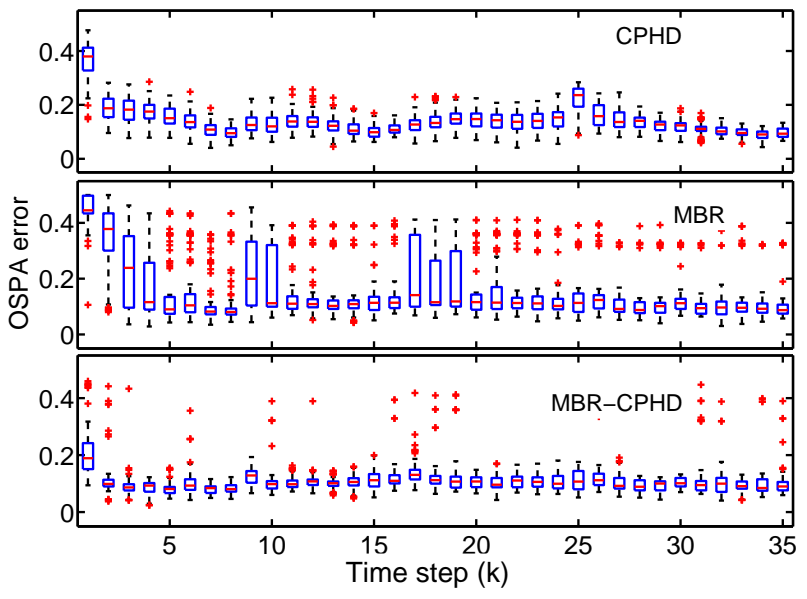

(b) Exp. 2 Box-and-whisker plot

Figure 3: (a) True cardinality and (b) box-and-whisker plot for Exp. 2. Boxes indicate the 25-75 interquartile range; whiskers extend 1.5 times the range and ' + ' symbols indicate outliers lying beyond the whiskers.

\section{CONCLUSIONS}

We developed the hybrid multi-Bernoulli CPHD filter for superpositional sensors. An IIDC RFS is introduced in the prediction step to account for newborn targets. The update step propagates the conditional PHD for each component of the RFS. Update equation for the cardinality distribution of the IIDC RFS is also given. Finally the new targets identified by the IIDC RFS component are approximated by Bernoulli components so that each target has its own density function which in turn leads to better state estimation. The hybrid filter has been compared with the multi-Bernoulli and CPHD filter in a simulated multitarget tracking application using the radio frequency measurement modality. From simulations it can be seen that the hybrid filter is more accurate than the multi-Bernoulli filter; while the cardinality estimate is not as robust as that of the CPHD filter, the discrepancy is not substantial, and the state estimates of individual targets are more accurate. The hybrid multi-Bernoulli CPHD filter is also computationally faster than the multi-Bernoulli or CPHD filter. 


\section{REFERENCES}

1. R. Mahler, "Statistics 101" for multisensor, multitarget data fusion," IEEE Aerospace and Electronic Systems Magazine 19(1), pp. 53-64, 2004.

2. R. Mahler, Statistical multisource-multitarget information fusion, Artech House, Boston, 2007.

3. R. Mahler, "CPHD filters for superpositional sensors," in Proc. SPIE Signal and Data Proc. for Small Targets, 7445, (San Diego, CA, U.S.A.), Aug. 2009.

4. B. Balakumar, A. Sinha, T. Kirubarajan, and J. Reilly, "PHD filtering for tracking an unknown number of sources using an array of sensors," in Proc. Workshop Stat. Sig. Proc., (Bordeaux, France), Jul. 2005.

5. D. Angelosante, E. Biglieri, and M. Lops, "Sequential estimation of multipath MIMO-OFDM channels," IEEE Trans. Sig. Proc. 57(8), pp. 3167-3181, 2009.

6. O. Hlinka, O. Sluciak, F. Hlawatsch, P. M. Djuric, and M. Rupp, "Likelihood consensus and its application to distributed particle filtering," IEEE Trans. Sig. Proc. 60(8), pp. 4334-4349, 2012.

7. J. Wilson and N. Patwari, "Radio tomographic imaging with wireless networks," IEEE Trans. Mobile Computing 9(5), pp. 621-632, 2010.

8. F. Thouin, S. Nannuru, and M. Coates, "Multi-target tracking for measurement models with additive contributions," in Proc. Int. Conf. Information Fusion, (Chicago, IL, U.S.A.), Jul. 2011.

9. F. Thouin, S. Nannuru, and M. Coates, "Multi-target tracking for measurement models with additive contributions," http://networks.ece.mcgill.ca/node/189.

10. R. Mahler and A. El-Fallah, "An approximate CPHD filter for superpositional sensors," in Proc. SPIE Int. Conf. Sig. Proc., Sensor Fusion, Target Recog., (Baltimore, MD, U.S.A.), Apr. 2012.

11. S. Nannuru, M. Coates, and R. Mahler, "Computationally-tractable approximate PHD and CPHD filters for superpositional sensors," IEEE J. Sel. Topics in Sig. Proc. 7(3), pp. 410-420, 2013.

12. D. Hauschildt, "Gaussian mixture implementation of the cardinalized probability hypothesis density filter for superpositional sensors," in Proc. Int. Conf. Indoor Positioning and Indoor Navigation, (Guimaraes, Portugal), Sep. 2011.

13. S. Nannuru and M. Coates, "Multi-Bernoulli filter for superpositional sensors," in Proc. Int. Conf. Inf. Fusion, (Istanbul, Turkey), Jul. 2013.

14. S. Nannuru and M. Coates, "Particle filter implementation of the multi-Bernoulli filter for superpositional sensors," in 5th Int. Workshop on Comp. Advances in Multi-Sensor Adaptive Proc., (Saint Martin), Dec. 2013.

15. J. L. Williams, "Hybrid Poisson and multi-Bernoulli filters," in Proc. Int. Conf. Information Fusion, (Singapore), Jul. 2012.

16. J. L. Williams, "Marginal multi-Bernoulli filters (extended version)," ArXiv e-prints, http://arxiv.org/abs/1203.2995, Mar. 2012.

17. E. Pollard, B. Pannetier, and M. Rombaut, "Hybrid algorithms for multitarget tracking using MHT and GM-CPHD," IEEE Trans. Aerospace and Electronic Systems 47(2), pp. 832-847, 2011.

18. K. Panta, B.-N. Vo, and S. Singh, "Novel data association schemes for the probability hypothesis density filter," IEEE Trans. Aerospace and Electronic Systems 43(2), pp. 556-570, 2007.

19. S. Nannuru and M. Coates, "Technical report: Multi-Bernoulli filter and hybrid multi-Bernoulli CPHD filter for superpositional sensors," http://networks.ece.mcgill.ca/node/306 , 2014.

20. B. Vo, B. Vo, N. Pham, and D. Suter, "Joint detection and estimation of multiple objects from image observations," IEEE Trans. Signal Proc. 58(10), pp. 5129-5141, 2010.

21. B.-N. Vo, S. Singh, and A. Doucet, "Sequential Monte Carlo methods for multitarget filtering with random finite sets," IEEE Trans. Aerospace and Electronic Systems 41(4), pp. 1224-1245, 2005.

22. S. Nannuru, Y. Li, M. Coates, and B. Yang, "Multi-target device-free tracking using radio frequency tomography," in Proc. Int. Conf. Intelligent Sensors, Sensor Networks and Information Processing, (Adelaide, Australia), Dec. 2011.

23. S. Nannuru, Y. Li, Y. Zeng, M. Coates, and B. Yang, "Radio frequency tomography for passive indoor multi-target tracking," IEEE Trans. Mobile Computing 12(12), pp. 2322-2333, 2013.

24. D. Schuhmacher, B.-T. Vo, and B.-N. Vo, "A consistent metric for performance evaluation of multi-object filters," IEEE Trans. Signal Proc. 56(8), pp. 3447-3457, 2008. 